\title{
Estado nutricional de niños palúdicos residentes en El Bagre y Turbo, Antioquia, Colombia, 2004-2005
}

\author{
Rosa Magdalena Uscátegui', Adriana María Correa² \\ ${ }^{1}$ Grupo de Investigación en Alimentación y Nutrición Humana, Escuela de Nutrición y Dietética, Universidad \\ de Antioquia, Medellín, Colombia \\ ${ }^{2}$ Grupo de Malaria, Facultad de Medicina, Universidad de Antioquia, Medellín, Colombia
}

Introducción. El paludismo, o malaria, y la desnutrición coexisten en las regiones más pobres del mundo. En las zonas colombianas donde el paludismo es endémico, la desnutrición también constituye un problema de salud pública.

Objetivo. Determinar la prevalencia de desnutrición de niños palúdicos de 4 a 10 años de edad e identificar algunos de sus factores condicionantes.

Materiales y métodos. Se evaluaron 93 niños palúdicos de los municipios de Turbo y El Bagre, Antioquia, Colombia, participantes en un estudio experimental, balanceado, no ciego, para evaluar su respuesta al tratamiento antipalúdico administrado junto con un suplemento de vitamina $\mathrm{A}$ y antiparasitarios en dosis única. Al ingresar al estudio se evaluó el estado nutricional por antropometría y se realizó una encuesta sobre las condiciones sociales y económicas de las familias de estos niños.

Resultados. La prevalencia de niños con desnutrición crónica moderada o grave fue de 22,6\%; la de desnutrición global moderada o grave de 10,8\% y la aguda de 2,3\%. Las prevalencias fueron mayores entre varones y residentes en Turbo.

Conclusiones. Las prevalencias de desnutrición fueron superiores a las reportadas en la última Encuesta Nacional de Demografía y Salud (2005), y en los residentes en Turbo fueron mayores a las encontradas en el Urabá antioqueño.

Palabras clave: estado nutricional, paludismo/terapia, niño, factores socioeconómicas, antropometría, Colombia

\section{Nutritional status of children with malaria in northwest Colombia}

Introduction. Malaria and malnutrition coexist within the poorest regions of the world. In the regions of Colombia where malaria is endemic, malnutrition is also a public health problem. Objective. The prevalence of malnutrition in children with malaria was determined and several factors associated with malnutrition were identified.

Materials and methods. A total of 93 children with malaria were included. They resided in the municipalities of Turbo and El Bagre (Antioquia, Colombia) and volunteered for an experimental, study to evaluate the outcome of an antimalarial treatment with a vitamin A supplement and an anti-intestinal parasite treatment administered as a single dose. At enrollment, the nutritional status was evaluated by anthropometry, and a survey questionnaire was administered about economic and social conditions of the family.

Results. Prevalence of moderate or severe chronic malnutrition was $22.6 \%-10.8 \%$ of children had moderate or severe global malnutrition and $2.3 \%$ had acute malnutrition. The prevalence of malnutrition was higher in males and in children from Turbo.

Conclusions. Prevalence of malnutrition was higher than previously reported by the National Health and Demography Survey (2005) and, in Turbo, the prevalence was higher than that reported for the rest of the Urabá region of Antioquia.

Key words: nutritional status, malaria/therapy, child, socioeconomic factors, anthropometry, Colombia 
La desnutrición y la pobreza coexisten en las zonas endémicas de malaria (1), al mismo tiempo que el hambre es una fiel representación de la pobreza. Estos problemas, además de aumentar el riesgo de enfermar y morir, limitan el desarrollo físico y mental de los niños que los padecen. La pobreza es un flagelo que azota a más de 4.000 millones de personas en nuestro planeta y se convierte en un factor condicionante del estilo de vida que determina la salud individual y colectiva (2). El paludismo, o malaria, es una de las enfermedades asociadas con la pobreza; a partir de 1960 la incidencia se redujo en muchos países, pero alrededor de 1970 comenzó su resurgimiento. Los países más afectados en América Latina son Brasil, Perú, Colombia, Honduras y Nicaragua (3).

En Colombia durante el año 2002 se presentaron 195.719 casos entre 22 ' 403.000 personas expuestas, con un índice parasitario anual (IPA: número de casos positivos para paludismo por mil personas expuestas) ajustado de 8,74 por mil, con 89.753 casos por Plasmodium falciparum o por esta y otra especie (malaria mixta), 105.931 casos por $P$. vivax y 35 casos por $P$. malariae. Durante el mismo año, la mortalidad por paludismo en toda América fue de 168 personas, de las cuales, 40 (24\%) residían en Colombia (4). En Urabá y Bajo Cauca, regiones que aportan más de $90 \%$ de los casos de paludismo en el departamento de Antioquia, se registraron índices parasitarios anuales promedio de 39 en Turbo y 156 en El Bagre-Zaragoza en el período 1996-2000 $(1,5)$.

Otro problema con alta prevalencia entre los niños de Turbo y El Bagre es la desnutrición; de acuerdo con el estudio "Perfil alimentario y nutricional de los hogares de Antioquia" realizado en 2005 en la región del Bajo Cauca, donde se ubica el municipio de El Bagre, se encontró que entre los niños

\footnotetext{
Correspondencia:

Rosa Magdalena Uscátegui, Calle 62 № 52-59, laboratorio 413, apartado aéreo 1226, SIU, Universidad de Antioquia, Medellín, Colombia.

Teléfono: (054) 2106498 ; fax: 2106487 y 2106400 , extensión 3352

rosauscategui@gmail.com
}

Recibido: 03/05/07; aceptado: 03/09/07 menores de 10 años, 27,5\% presentaba desnutrición crónica (talla para la edad<-2 unidades Z), 19,2\% tenía desnutrición global (peso para la edad $<-2$ unidades $Z$ ) y 3,3\% desnutrición aguda (peso para la talla $<-2$ unidades Z) (6). Este mismo estudio reveló que para el Urabá antioqueño, donde se encuentra el municipio de Turbo, $20,7 \%$ de los niños presentaban desnutrición crónica, $14,4 \%$ global y $3,4 \%$ aguda (7).

En las dos regiones y para los tres tipos de desnutrición, las prevalencias fueron muy superiores a las reportadas en la última Encuesta Nacional de Demografía y Salud para los niños entre 5 y 9 años de edad, de los cuales, $13 \%$ presentaba desnutrición crónica, $5 \%$ global y $1 \%$ aguda (8).

Estudios anteriores en población palúdica, en quienes se evaluó el riesgo de desnutrición (<-1 unidad Z) para los diferentes indicadores antropométricos, revelaron que en El Bagre el riesgo de desnutrición crónica en los niños de 4 a 9 años de edad era de $63 \%$, el de aguda, $8 \%$ y el de global, $47 \%$ (9). En otro estudio en el que se incluyeron 128 niños de 3 a 11 años, 66 residentes en El Bagre y Zaragoza y 62 en Turbo, 33\% presentó riesgo de desnutrición aguda, $52 \%$ de desnutrición crónica, y $56 \%$ de desnutrición global. El $67 \%$ de los niños presentaba riesgo de desnutrición de alguna clase y, de ellos, 54\% estaba en Turbo. El riesgo de desnutrición crónica fue mayor en Turbo (63\%), frente a $42 \%$ en El Bagre, pero en este último el riesgo de desnutrición aguda fue superior, $38 \%$ versus $28 \%$ (10).

El estado nutricional depende directamente de la disponibilidad de alimentos, así como del consumo y el aprovechamiento biológico de los mismos, factores que a su vez están determinados por las condiciones sociales y económicas. La pobreza hace vulnerables social y biológicamente a los individuos; una de sus consecuencias biológicas es la baja estatura de la población infantil (11). De ahí, la importancia de explorar las condiciones socioeconómicas de las familias cuando se evalúa el estado nutricional de los niños.

Este artículo forma parte de una investigación realizada en niños palúdicos sobre el efecto en la respuesta terapéutica antipalúdica del suplemento 
con vitamina $A$ oral y el tratamiento antihelmíntico y antiprotozoario de dosis única, administrados concomitantemente. Se evaluaron el estado nutricional de los niños por los indicadores peso para la edad, peso para la talla y talla para la edad y algunas condiciones socioeconómicas de las familias con el objetivo de determinar la prevalencia de desnutrición en niños palúdicos de 4 a 10 años de edad e identificar algunos de sus factores condicionantes.

\section{Materiales y métodos}

\section{Clase de estudio}

Se conformaron cuatro grupos de niños (25 por grupo) para evaluar el papel del suplemento con vitamina A y el tratamiento antiparasitario intestinal (albendazol y secnidazol) en la respuesta terapéutica antipalúdica. Los cuatro grupos se evaluaron simultáneamente mediante un diseño experimental, balanceado, con asignación aleatoria al grupo, con determinación no ciega del efecto (la respuesta terapéutica). En el estudio participaron 93 pacientes, 11 de ellos con malaria por $P$. falciparum y 82 con $P$. vivax.

\section{Unidad de análisis y diseño de la muestra}

La unidad de análisis fue cada niño con malaria no complicada según los criterios de la Organización Mundial de la Salud (OMS) (12), y sometido a tratamiento antipalúdico estándar. En 2000-2003 en Turbo y en El Bagre se presentaron 12.729 casos de paludismo en niños de 5 a 14 años, es decir, un promedio de 3.182 casos por año. Debido a que no se contaba con el número de casos en los niños de 4 a 10 años, se trabajó con este promedio. El nivel de confianza fue del $95 \%$ y el error de muestreo de $10 \%$.

El fenómeno que interesaba estudiar era múltiple según nuestro objetivo y la prevalencia de cada uno variaba, así: prevalencia de falla del tratamiento antipalúdico estimada en $10 \%$, de parásitos intestinales estimable en $80 \%$, de desnutrición crónica (peso para la talla<-1 unidad Z) $65 \%$ y de pobreza económica, $60 \%$. Se decidió trabajar con una proporción de $50 \%$ para forzar una muestra de máximo tamaño y garantizar que fuera apropiada para todos los fenómenos. Se obtuvo un tamaño de 93 que se aproximó a 100 personas y se decidió estudiar 50 niños en El Bagre y 50 en Turbo.

Los pacientes se captaron por orden de llegada a los puestos de malaria en la zona urbana de El Bagre y Turbo, a donde acudieron por su iniciativa y fueron incluidos en el estudio si satisfacían los criterios de inclusión y sus acudientes daban el consentimiento informado por escrito. La captación se hizo entre julio de 2004 y julio de 2005 y a cada niño se le hizo seguimiento durante 30 días, con varias evaluaciones en ese lapso.

\section{Población de referencia}

La población de referencia la integraron personas de 4 a 10 años de cualquier sexo, residentes en las zonas urbanas o rurales de los municipios de El Bagre y Turbo, que consultaron al puesto de diagnóstico de malaria de cada lugar por presentar síndrome febril y a quienes se les demostró mediante examen de gota gruesa infección palúdica.

\section{Criterios de inclusión en el estudio}

Los pacientes con paludismo se admitieron si cumplían todos estos requisitos:

a) tener paludismo por $P$. vivax o $P$. falciparum, sin incluir infecciones mixtas;

b) tener parasitemia entre 1.000 y 50.000 parásitos asexuales por microlitro;

c) residir en El Bagre o en Turbo, bien fuera en área rural o urbana;

d) tener entre 4 y 10 años de edad;

e) acudir voluntariamente al puesto de diagnóstico de malaria;

f) no tener enfermedad aparente distinta del paludismo;

g) no tener paludismo complicado, según los criterios de la OMS (12);

h) aceptar participar en el estudio mediante consentimiento informado y escrito firmado por su acudiente;

i) no haber tenido paludismo en los 30 días previos. 


\section{Criterios de exclusión en el estudio}

a) presentar alguna otra enfermedad, trauma, accidente o envenenamiento;

b) no cumplir con las citas para evaluación los días 2, 4, 7-8, 14-15 y 28-31.

\section{Evaluación nutricional}

El peso fue tomado de pie, sin zapatos y con el mínimo de ropa; se utilizó una báscula electrónica de $100 \mathrm{~kg}$ de capacidad y $0,01 \mathrm{~kg}$ de sensibilidad. La talla se midió de pie, sin zapatos y con tallímetro flexible fijo en la pared, que tenía $2 \mathrm{~m}$ de capacidad y $1 \mathrm{~mm}$ de sensibilidad. Las mediciones las realizaron los dos médicos participantes en el estudio, previa capacitación y estandarización en técnicas antropométricas de uso internacional (13). Cada medida se evaluó y se registró en el formulario dos veces y se trabajó con el promedio de las mismas. La edad se calculó por diferencia entre la fecha de nacimiento y la de la evaluación.

La evaluación del estado nutricional se hizo comparando la población en estudio con el patrón tipo, establecido como población de referencia por el National Center for Health Statistics de los Estados Unidos (NCHS) en 1977, aceptado por la OMS para comparaciones internacionales (14). Con el peso, la talla, la edad y el sexo, se construyeron los siguientes índices: talla para la edad, que mide desnutrición crónica (retardo en el crecimiento lineal); peso para la edad, que refleja la desnutrición global (o general) y, finalmente, peso para la talla, que permite evaluar la desnutrición aguda (11).

En el cuadro 1 se resume el sistema de clasificación para cada uno de los indicadores.

\section{Condiciones socioeconómicas y otras evaluaciones}

A la persona que llevó al niño a la consulta se le diligenció un formulario para evaluar las condiciones sociales y económicas de la familia, en el que se indagó por el ingreso familiar, el aporte para alimentación, el número de miembros de la familia y el de personas que duermen en la casa, número de personas por habitación y disponibilidad de servicios públicos.

A los niños se les realizó un examen coprológico por concentración en formol-éter al inicio del estudio, a los 8 días y a los 30 . Se consideró como positiva la presencia de protozoarios patógenos o huevos o larvas de helmintos; en caso contrario, se clasificó como negativo.

\section{Análisis de los datos}

Los datos se procesaron y analizaron en los programas Epi-Info versión 6.04D (Centers for Disease Control and Prevention, Atlanta, Georgia, United States) y SPSS, versión 14. La comparación de los valores de los indicadores talla para la edad, peso para la edad y peso para la talla en unidades $Z$, según características sociodemográficas y clínicas, se hizo por la prueba $U$ de Mann-Whitney, lo mismo que la comparación de los valores de las variables económicas y familiares, entre desnutridos y no desnutridos, para los diferentes indicadores antropométricos. La comparación de la proporción de desnutridos según municipio y sexo, se hizo por la prueba de diferencia de proporciones. La comparación de la cantidad mensual promedio invertida en alimentación entre las zonas urbana y rural de cada municipio y entre los dos

Cuadro 1. Categorías empleadas para clasificar el estado nutricional.

\begin{tabular}{llll}
\hline $\begin{array}{l}\text { Punto de corte } \\
\text { unidades } \mathbf{Z}\end{array}$ & & Clasificación según unidades $\mathbf{Z}$ & \\
& Talla para la edad & Peso para la edad & Peso para la talla \\
\hline$>2,00$ & Alto & Exceso & Obesidad \\
1,01 a 2,00 & Alto & Exceso & Sobrepeso \\
$-1,00$ a 1,00 & Normal & Normal & Normal \\
$-1,01$ a $-2,00$ & Desnutrición crónica leve & Desnutrición global leve & Desnutrición aguda leve \\
$-2,01$ a $-3,00$ & Desnutrición crónica moderada & Desnutrición global moderada & Desnutrición aguda moderada \\
$<-3,00$ & Desnutrición crónica grave & Desnutrición global grave & Desnutrición aguda grave \\
\hline
\end{tabular}


municipios, se hizo mediante la prueba de KruskalWallis. En todos los casos se aceptó como significativo un valor de $p<0,05$.

\section{Consideraciones éticas}

El estudio fue avalado por el Comité de Ética del Centro de Investigaciones Médicas de la Universidad de Antioquia. Cada acudiente del niño recibió explicación detallada del proyecto y firmó el consentimiento informado escrito.

\section{Resultados}

Participaron en el estudio 93 niños, con edades entre 4 y 10 años (promedio \pm desviación estándar: $7 \pm 2$ ), similar entre los niños de Turbo y los de El Bagre. El promedio de peso fue $21,6 \pm 5,4 \mathrm{~kg}$ y el de talla $117,5 \pm 12,9 \mathrm{~cm}$, sin diferencias estadísticamente significativas entre los municipios.

En el cuadro 2 se presentan algunas características sociodemográficas, clínicas y de salud ambiental; igualmente se incluyen los valores de $p$ de la comparación de las medias en unidades $Z$ de los indicadores talla para la edad, peso para la edad y peso para la talla entre los municipios de procedencia, el sexo, la presencia de parásitos intestinales, especie de Plasmodium y la disponibilidad de servicios públicos. En Turbo se evaluaron 48 niños (47 familias) y en El Bagre 45 (41 familias), de los cuales, 60 eran hombres y 33 mujeres. En la zona rural residía $58 \%$ de los niños y la distribución por zona fue muy diferente en cada municipio: en El Bagre vivía $44 \%$ de los niños en la zona rural y en Turbo $74 \%(p=0,003)$.
El $89 \%$ de los niños tenía paludismo por $P$. vivax y el resto por $P$. falciparum. El agua para beber y cocinar se obtenía de acueducto únicamente en las familias de $30 \%$ de los niños, sólo $42 \%$ de ellas poseían sanitario en su vivienda y $29 \%$ disponía de servicio de recolección de basuras.

Los indicadores antropométricos en unidades $Z$ no mostraron diferencias según la disponibilidad de servicios sanitarios, acueducto y recolección de basuras, excepto para el indicador talla para la edad que fue estadísticamente menor entre quienes no disponían de agua de acueducto, en comparación con quienes sí tenían este servicio $(p=0,038)$; también fue más bajo entre aquéllos que no tenían servicio sanitario $(p=0,044)$.

Los indicadores peso para la edad y peso para la talla fueron significativamente menores en los niños del municipio de Turbo, $p=0,040$ y $p=0,002$, respectivamente. En los tres indicadores antropométricos estudiados, no hubo diferencias según sexo, presencia de parásitos gastrointestinales, ni la especie de Plasmodium causante del paludismo (cuadro 2).

\section{Resultados de la evaluación nutricional}

La desnutrición global (peso para la edad) fue $46,2 \%$ en el grupo total, con predominio de la desnutrición global leve $(35,5 \%)(<-1$ a -2 unidades Z) y menor proporción de sujetos en las categorías moderada y grave (<-2 unidades Z) que en conjunto sumaron $10,8 \%$. Los únicos dos casos de desnutrición global grave se presentaron en Turbo, mientras que los dos con

Cuadro 2. Características demográficas, clínicas y de salud ambiental y su relación con los valores de talla para la edad, peso para la edad y peso para la talla en unidades $Z$.

\begin{tabular}{lcccc}
\hline Características & $\mathbf{n}$ & $\mathbf{T / E} \mathbf{1}^{\mathbf{1}}$ & $\mathbf{P / \mathbf { E } ^ { 1 }}$ & $\mathbf{P}^{\mathbf{1}}$ \\
\hline Municipio (El Bagre/Turbo) & $48 / 45$ & 0,175 & $\mathbf{0 , 0 4 0}$ & $\mathbf{0 , 0 0 2}$ \\
Sexo (hombres/mujeres) & $60 / 33$ & 0,659 & 0,492 & 0,960 \\
Coprológico (positivo/negativo) & $72 / 13$ & 0,347 & 0,410 & 0,281 \\
Especie de Plasmodium (vivax/ falciparum) & $82 / 11$ & 0,521 & 0,212 & 0,110 \\
Sanitario (Sí/No) & $37 / 50$ & $\mathbf{0 , 0 4 4}$ & 0,370 & 0,110 \\
Agua de acueducto (Sí/No) & $24 / 57$ & $\mathbf{0 , 0 3 8}$ & 0,247 & 0,899 \\
Recolección de basuras (Sí/No) & $26 / 65$ & 0,735 & 0,782 & 0,920 \\
\hline
\end{tabular}

${ }^{1}$ Valor de $p$ de la comparación de las medianas en unidades $Z$, de los indicadores T/E (talla para la edad), P/E (peso para la edad) y $\mathrm{P} / \mathrm{T}$ (peso para la talla), según diversas características sociodemográficas y clínicas, por la prueba U de MannWhitney. En negrilla se resaltan los valores con $p<0,05$. 
exceso de peso, correspondían a niños residentes en El Bagre. Sin embargo, no se presentaron diferencias estadísticamente significativas según zona de residencia de los niños. Aunque la prevalencia de desnutrición global fue similar por sexo $(p=0,585)$, las mujeres presentaron mayor prevalencia de desnutrición moderada a grave (cuadro 3 ).

En el grupo total la prevalencia de desnutrición aguda fue de 14,9\%; la mayor proporción estaba en la categoría leve $(12,6 \%)$ y aunque no se presentaron casos de desnutrición grave, 2,3\% tenían desnutrición aguda moderada $(<-2$ y -3 unidades Z). El porcentaje total de niños con desnutrición aguda fue significativamente mayor en Turbo $(p=0,019)$; de hecho, los únicos dos niños que presentaron desnutrición moderada residían en este municipio, mientras que los tres que tenían sobrepeso u obesidad por el indicador peso para la talla eran residentes de El Bagre. No hubo diferencias estadísticamente significativas en la proporción de desnutrición aguda entre hombres y mujeres $(p=0,319)$ (cuadro 4 ).

El 53,8\% de los niños estudiados tenía algún grado de desnutrición crónica (baja talla para la edad), $44,1 \%$ tenían talla adecuada y solamente $2,2 \%$ tenían talla alta. La proporción de niños con desnutrición crónica fue de $57,8 \%$ en Turbo y $50,0 \%$ en El Bagre, sin diferencias estadísticamente significativas $(p=0,452)$; tampoco se observó diferencia por sexo ( $p=0,449)$ (cuadro 5). El 22,6\% de los niños tenía desnutrición crónica entre moderada y grave (<-2 unidades $Z$ ).

\section{Relación entre características socioeconómicas y desnutrición}

El ingreso promedio de los miembros de la familia que trabajaban fue de $\$ 285.090 \pm \$ 187.344$ por mes y su aporte a los gastos familiares para

Cuadro 3. Clasificación nutricional por el indicador peso para la edad, según lugar de procedencia y sexo.

\begin{tabular}{|c|c|c|c|c|c|}
\hline \multirow[b]{2}{*}{ Clasificación peso para la edad } & \multirow[b]{2}{*}{$\begin{array}{c}\text { Total } \\
(\mathrm{n}=93) \\
\%\end{array}$} & \multicolumn{2}{|c|}{ Procedencia } & \multicolumn{2}{|c|}{ Sexo } \\
\hline & & $\begin{array}{c}\text { El Bagre } \\
(n=48) \\
\%\end{array}$ & $\begin{array}{c}\text { Turbo } \\
(n=45) \\
\%\end{array}$ & $\begin{array}{c}\text { Hombres } \\
(n=60) \\
\%\end{array}$ & $\begin{array}{c}\text { Mujeres } \\
\begin{array}{c}(\mathrm{n}=33) \\
\%\end{array}\end{array}$ \\
\hline Desnutrición global grave & 2,2 & 0,0 & 4,4 & 1,7 & 3,0 \\
\hline Desnutrición global moderada & 8,5 & 4,2 & 13,3 & 6,7 & 12,1 \\
\hline Desnutrición global leve & 35,5 & 35,4 & 35,6 & 40,0 & 27,3 \\
\hline Desnutrición total & 46,2 & 39,6 & $53,3^{1}$ & 48,4 & $42,4^{2}$ \\
\hline Adecuado & 51,6 & 56,2 & 46,7 & 48,3 & 57,6 \\
\hline Exceso de peso & 2,2 & 4,2 & 0,0 & 3,3 & 0,0 \\
\hline Total & 100 & 100 & 100 & 100 & 100 \\
\hline
\end{tabular}

1 Comparación de prevalencia de desnutrición total según procedencia, $p=0,184$

${ }^{2}$ Comparación de prevalencia de desnutrición total según sexo, $p=0,585$

Cuadro 4. Clasificación nutricional por el indicador peso para la talla, según lugar de procedencia y sexo.

\begin{tabular}{|c|c|c|c|c|c|}
\hline \multirow[b]{2}{*}{ Clasificación peso para la talla } & \multirow[b]{2}{*}{$\begin{array}{c}\text { Total } \\
(\mathrm{n}=87) \\
\%\end{array}$} & \multicolumn{2}{|c|}{ Procedencia } & \multicolumn{2}{|c|}{ Sexo } \\
\hline & & $\begin{array}{c}\text { El Bagre } \\
(\mathrm{n}=46) \\
\%\end{array}$ & $\begin{array}{c}\text { Turbo } \\
(n=41) \\
\%\end{array}$ & $\begin{array}{c}\text { Hombres } \\
(n=60) \\
\%\end{array}$ & $\begin{array}{c}\text { Mujeres } \\
(n=27) \\
\%\end{array}$ \\
\hline Desnutrición aguda moderada & 2,3 & 0,0 & 4,9 & 1,7 & 3,7 \\
\hline Desnutrición aguda leve & 12,6 & 6,5 & 19,5 & 16,6 & 3,7 \\
\hline Desnutrición total & 14,9 & 6,5 & $24,4^{1}$ & 18,3 & $7,4^{2}$ \\
\hline Normal & 81,7 & 87,0 & 75,6 & 76,7 & 92,6 \\
\hline Sobrepeso & 2,3 & 4,3 & 0,0 & 3,3 & 0,0 \\
\hline Obesidad & 1,1 & 2,2 & 0,0 & 1,7 & 0,0 \\
\hline Total & 100 & 100 & 100 & 100 & 100 \\
\hline
\end{tabular}

${ }^{1}$ Comparación de prevalencia de desnutrición total según procedencia, $\mathrm{p}=0,019$

${ }^{2}$ Comparación de prevalencia de desnutrición total según sexo, $p=0,319$ 
alimentación fue $\$ 258.000$ pesos por mes, equivalente al $90 \%$ de los ingresos. Los promedios devengados fueron similares por municipio $(p=$ $0,357)$ y entre la zona urbana y la rural $(p=0,121)$. La suma mensual promedio invertida en alimentación fue mayor en El Bagre $(\$ 269.000 \pm$ $\$ 133.000)$ que en Turbo $(\$ 151.000 \pm \$ 99.000)$ $(p=0,00000)$, pero no hubo diferencias entre la zona urbana y la rural $(p=0,376)$.

Se compararon los promedios entre desnutridos y no desnutridos, para los tipos de desnutrición crónica, aguda y global, de los ingresos familiares, el aporte para alimentación, el número de miembros de la familia, las personas que duermen en la casa y el número de personas por habitación; los valores de $p$ de dichas comparaciones se presentan en el cuadro 6. Los ingresos de las familias de los niños desnutridos y los no desnutridos, de acuerdo con los diferentes indicadores antropométricos, no presentaron diferencias estadísticamente significativas. En promedio, las familias estaban integradas por 6,4 $\pm 2,1$ miembros, el número de personas que dormía en la casa fue de $7,0 \pm 3,0$ y el de personas por cuarto de $3,4 \pm 1,6$. El número de integrantes de la familia y de personas que duermen en la casa fueron significativamente mayores entre los niños con desnutrición crónica y entre aquéllos con desnutrición aguda, quienes también tenían mayor número de personas por habitación (cuadro 6).

\section{Discusión}

La comparación del peso y la talla de los niños de nuestro estudio con la población de referencia, muestra que se desvía hacia la izquierda, lo que

Cuadro 5. Clasificación nutricional por el indicador talla para la edad, según lugar de procedencia y sexo.

\begin{tabular}{|c|c|c|c|c|c|}
\hline \multirow[b]{2}{*}{ Clasificación talla para la edad } & \multirow[b]{2}{*}{$\begin{array}{c}\text { Total } \\
(n=93) \\
\%\end{array}$} & \multicolumn{2}{|c|}{ Procedencia } & \multicolumn{2}{|c|}{ Sexo } \\
\hline & & $\begin{array}{c}\text { El Bagre } \\
(n=48) \\
\%\end{array}$ & $\begin{array}{c}\text { Turbo } \\
(n=45) \\
\%\end{array}$ & $\begin{array}{c}\text { Hombres } \\
(n=60) \\
\%\end{array}$ & $\begin{array}{c}\text { Mujeres } \\
(n=33) \\
\%\end{array}$ \\
\hline Desnutrición crónica grave & 5,4 & 2,1 & 8,9 & 1,7 & 12,1 \\
\hline Desnutrición crónica moderada & 17,2 & 14,6 & 20,0 & 21,7 & 9,1 \\
\hline Desnutrición crónica leve & 31,2 & 33,3 & 28,9 & 33,3 & 27,3 \\
\hline Desnutrición total & 53,8 & 50,0 & $57,8^{1}$ & 56,7 & $48,5^{2}$ \\
\hline Adecuado & 44,1 & 47,9 & 40,0 & 41,6 & 48,5 \\
\hline Normal alto & 2,1 & 2,1 & 2,2 & 1,7 & 3,0 \\
\hline Total & 100 & 100 & 100 & 100 & 100 \\
\hline
\end{tabular}

${ }^{1}$ Comparación de prevalencia de desnutrición total según procedencia, $p=0,452$

${ }^{2}$ Comparación de prevalencia de desnutrición total según sexo, $p=0,449$

Cuadro 6. Comparación de algunas características económicas y familiares, según estado nutricional, por los indicadores talla para la edad, peso para la edad y peso para la talla.

\begin{tabular}{|c|c|c|c|c|}
\hline Variable & $\bar{X} \pm D E$ & $\begin{array}{c}\text { Des/no Des } \\
T / E^{(1)}\end{array}$ & $\begin{array}{c}\text { Des/no Des } \\
\text { P/E }\end{array}$ & $\begin{array}{c}\text { Des/no Des } \\
\mathbf{P} / \mathbf{T}^{(1)}\end{array}$ \\
\hline Ingreso familiar (miles de pesos) & $285 \pm 187$ & 0,791 & 0,541 & 0,429 \\
\hline Aporte para alimentación (miles de pesos) & $258 \pm 155$ & 0,853 & 0,586 & 0,457 \\
\hline Miembros de la familia $(n)$ & $6,4 \pm 2,1$ & 0,024 & 0,653 & 0,015 \\
\hline Personas que duermen en la casa $(n)$ & $7,0 \pm 3,0$ & 0.024 & 0,615 & 0,012 \\
\hline Personas por habitación (n) & $3,4 \pm 1,6$ & 0,093 & 0,690 & 0,033 \\
\hline
\end{tabular}

$\overline{\mathrm{X}} \pm \mathrm{DE}$ : promedio \pm desviación estándar; Des: desnutrido (<-1 unidad Z); no Des: no desnutridos ( $\geq-1$ unidades Z).

${ }^{1}$ Valor de $p$ de la comparación del promedio de las variables económicas y familiares, entre desnutridos y no desnutridos para los indicadores T/E (talla para la edad), P/E (peso para la edad) y P/T (peso para la talla), por la prueba U de MannWhitney.

${ }^{2}$ En negrilla se resaltan los valores con $p<0,05$. 
podría explicarse o deberse a las precarias condiciones sociales, económicas y ambientales en que viven estas familias, por lo que es poco probable que esto se deba a factores genéticos, más que ambientales.

Se ha demostrado que las principales variaciones en el crecimiento físico entre poblaciones se deben a factores ambientales y socioeconómicos, más que a diferencias étnicas o raciales (15). Por ello, para la clasificación nutricional utilizamos un punto de corte por debajo de una desviación estándar (<-1 unidad Z) y hablamos de desnutrición leve, moderada e intensa, más que de riesgos de desnutrición (11). Aunque en la práctica se recomienda el uso de un punto crítico de $<-2$ unidades $Z$ para definir desnutrición en los programas de vigilancia nutricional de una población, hay que tener en cuenta que estos niños representan únicamente la punta del iceberg de la desnutrición porque sólo se identifican los casos de desnutrición grave (desnutrición moderada y grave) (16).

Pelletier y colaboradores, después de revisar 28 estudios prospectivos, concluyeron que el riesgo de mortalidad también es elevado entre los sujetos con desnutrición leve a moderada $(17,18)$. La desnutrición leve para los diferentes indicadores antropométricos, fue la categoría que agrupó la mayor proporción de sujetos en nuestro estudio. De hecho, la mortalidad relacionada con desnutrición en Colombia alcanza altas proporciones, especialmente entre los menores de cinco años, tal como lo establece el estudio "La mortalidad por desnutrición en Colombia, 19982002", en el que se encontró que una de cada cinco defunciones en niños colombianos entre 1 y 4 años, está asociada con la desnutrición (19).

Mientras la desnutrición infantil en Colombia ha disminuido durante los últimos 25 años, las cifras encontradas en nuestro estudio confirman que hay regiones geográficas donde el problema nutricional alcanza mayores proporciones. La prevalencia de desnutrición moderada y grave ( $<-2$ unidades $Z$ ) para los diferentes indicadores antropométricos en nuestro estudio, fueron cercanos al doble de la prevalencia nacional para la crónica y la global, pero fue más del triple de la prevalencia nacional para la desnutrición aguda (8). Inclusive son superiores a la prevalencia nacional de hace 16 años entre los menores de 5 años.

La desnutrición aguda, o déficit de peso para la estatura, corresponde en forma general a la depleción de la masa corporal y de la cantidad de reservas que se tienen en el organismo, depositadas como grasa. En casos de déficit acentuado se presenta pérdida de masa muscular y de otros tejidos (20).

El 46,2\% de los escolares estudiados presentó desnutrición global, cifra superior al promedio reportado para la población antioqueña de 6 a 10 años (42,0\%) (21) y fue el doble de la informada en la última Encuesta Nacional de Demografía y Salud (peso para la talla $<-2$ unidades Z) (8). Además del riesgo de enfermar y morir a que están expuestos los niños con bajo peso, este indicador ha mostrado capacidad pronóstica de mortalidad en la vida adulta por enfermedades crónicas degenerativas (11) que vienen en aumento en nuestro país y en la región de Urabá representan las primeras causas de mortalidad en adultos (22).

Más de la mitad $(53,8 \%)$ de los escolares con paludismo incluidos en nuestro estudio presentó desnutrición crónica. La estatura baja es en gran parte irreversible y tiene una consecuencia intergeneracional. Aunque el crecimiento físico posterior busque compensarlo, los niños de estatura baja siguen siendo más pequeños a lo largo de su vida y los cambios en la estatura pueden requerir varias generaciones. El problema va más allá de llegar a la vida adulta con baja estatura: lo que en realidad denota es que todo el cuerpo, incluido el cerebro, ha crecido por debajo de su potencial, hecho que pone en desventaja a estos niños.

De igual forma, la capacidad física de trabajo se relaciona con la estatura y con la cantidad de masa magra, lo que implica que los niños que tienen retardo en talla tendrán menor posibilidad de realizar un trabajo físico en la vida adulta, lo cual en zonas agrícolas y mineras poco tecnificadas como las regiones estudiadas, tendrá repercusiones sobre su productividad y sus ingresos económicos.

Los trabajos de Gerry Spurr y Julio César Reina (23-25), realizados en la década de los 80 , en población colombiana del Valle del Cauca, 
demostraron ampliamente que la desnutrición infantil deja un deterioro permanente en la capacidad física de trabajo y que, a su vez, esa reducción se relacionaba directamente con la baja productividad y los reducidos ingresos de los obreros que trabajaban para las empresas productoras de azúcar quienes recibían un salario proporcional a la cantidad de caña cortada manualmente. En los niños, la desnutrición y la enfermedad pueden tener consecuencias desastrosas que van desde la discapacidad que reduce las oportunidades futuras de desarrollo físico, mental y económico hasta la muerte (26).

El paludismo es un factor contribuyente al desarrollo de desnutrición, está bien documentado que las enfermedades infecciosas aumentan las necesidades nutricionales y se asocian con anorexia. Aunque no disponemos de información en población colombiana sobre este aspecto, los reportes en la década pasada de los estudios realizados en niños gambianos señalan que durante un episodio agudo de malaria, la tasa metabólica basal se incrementa entre $30 \%$ y $37 \%$, al mismo tiempo que el catabolismo proteico aumenta al doble $(27,28)$. Estudios recientes confirman que el desgaste muscular es un hallazgo frecuente entre las personas con paludismo (29).

Este incremento en las demandas energéticas y proteicas puede tener gran impacto sobre el estado nutricional de los niños residentes en las zonas estudiadas, si se tiene en cuenta que pueden sufrir varios episodios de paludismo al año.

En comparación con la prevalencia de desnutrición (valores <-1 unidad Z) reportada para la región de Urabá en el estudio "Perfil alimentario y nutricional de la población antioqueña" de 2005 (7), en los menores de 6 a 10 años, los niños de Turbo en nuestro estudio presentaron cifras de desnutrición global superiores $(53,3 \%$ Vs. $48,7 \%)$, cifras de desnutrición crónica similares con valores de $57,8 \%$ en los niños del presente estudio y $58,2 \%$ en la población estudiada por Álvarez (2005) (7), pero la prevalencia de desnutrición aguda fue más del doble en nuestro estudio $(24,4 \%$ frente a $11,5 \%)$.

Situación distinta de la descrita se presentó en los niños residentes en El Bagre, en donde la proporción de sujetos con valores <-1 unidad Z, para los diferentes indicadores antropométricos fue muy inferior a la encontrada en los niños de 6 a 10 años residentes en la región del Bajo Cauca antioqueño, estudiados en el perfil alimentario y nutricional de los hogares de Antioquia (6). Igualmente, en un estudio piloto con diseño prospectivo y transversal en 51 niños con edades entre 4 y 9 años que tenían paludismo por $P$. vivax (66\%), P. falciparum (30\%) o ambas (4\%) en comparación con 49 niños negativos para malaria, en el que se definió como riesgo de desnutrición tener valores inferiores a -1 unidad $Z$, se encontró un riesgo de desnutrición similar entre los palúdicos y quienes no tenían la enfermedad; $63 \%$ tuvo algún riesgo de desnutrición crónica, $7 \%$ algún riesgo de desnutrición aguda, y $49 \%$ algún riesgo de desnutrición global (9).

Esta diferencia se podría explicar porque los criterios para ingresar a nuestro estudio exigían que los niños asistieran a controles los días 1,2, $4,7-8,14-15$ y $28-31$ y las dificultades de acceso geográfico hicieron que los residentes en las zonas más alejadas del perímetro urbano con condiciones más precarias y de mayor aislamiento, donde la prevalencia de desnutrición probablemente sea mayor, no quedaran incluidos. Por esta misma razón, la proporción de niños del área rural de $\mathrm{El}$ Bagre fue muy inferior a la de Turbo.

En este estudio no se encontró asociación estadística entre la parasitemia y el estado nutricional, diferente a los hallazgos de Friedman y su grupo (30) en niños de 0 a 36 meses del occidente de Kenia, quienes encontraron mayores prevalencias de paludismo, anemia grave y alta parasitemia en los niños con desnutrición crónica en comparación con aquéllos sin este tipo de desnutrición.

Las diferencias entre nuestros hallazgos y los de Friedman podrían explicarse porque los grupos estudiados eran distintos: nuestros niños tenían mayor edad, menor prevalencia de desnutrición, predominio de paludismo por $P$. vivax y habitan zonas geográficas de transmisión inestable, mientras que en África oriental la transmisión de paludismo es intensa y predomina la malaria por P. falciparum. 
Las condiciones económicas de las familias de los niños participantes en el estudio son similares a las encontradas en otras investigaciones en Colombia, en las que la pobreza, en especial, la pobreza extrema o indigencia, se concentra en la zona rural. Cuando se mide a partir de los ingresos, más de las dos terceras partes de la población rural es pobre y más de la tercera parte viven en pobreza extrema (31). Esta situación ha presentado un crecimiento progresivo: de $68,4 \%$ de pobres entre la población rural en 1991 se pasó a $78,9 \%$ en 1997 , a $82,6 \%$ en 2000 y a $85,3 \%$ en 2003. En 2004, sólo $47 \%$ de la población rural lograba asegurar los ingresos requeridos para adquirir una canasta básica de alimentos (32).

El deterioro progresivo de las condiciones económicas del sector rural, que lleva al incremento de la pobreza, es en parte consecuencia de las políticas de apertura económica de los años noventa, impuestas a los países dependientes, que han arrasado con la agricultura nacional y llevado al hambre y la desnutrición (33). Otro factor que agrava la situación en la zona estudiada es el conflicto armado interno, con sus secuelas de destrucción de la base productiva de numerosos campesinos, más la violencia y el desplazamiento forzado de grandes grupos de población (34).

Las condiciones de saneamiento ambiental fueron precarias: la recolección de basuras, uno de los servicios públicos que causa mayor impacto sobre las condiciones del medio ambiente, sólo estaba disponible para $29 \%$ de las familias, proporción muy inferior a la reportada por el Departamento Administrativo Nacional de Estadística (DANE) según el censo de 2005 para el departamento de Antioquia (35). Igualmente, sólo el $30 \%$ de las familias estudiadas disponía de servicio de acueducto, cifra muy inferior a la publicada por la Organización Panamericana de la Salud en el informe del perfil de salud para Colombia, en el que se afirma que el $90 \%$ de la población colombiana dispone de agua potable (36).

Uno de los indicadores que mejor refleja las condiciones sociales y económicas de una población o un país es el crecimiento en talla $(37,38)$. De hecho, el indicador talla para la edad se relacionó con disponibilidad de agua de acueducto y servicio sanitario, lo mismo que con el tamaño de la familia y el número de personas que duermen en la casa, mientras que el indicador peso para la talla no se relacionó con la disponibilidad de servicios sanitarios, pero sí con el tamaño de la familia y con los indicadores de hacinamiento.

El promedio destinado a la alimentación para una familia de siete integrantes fue de $\$ 140.000$ mensuales, cifra muy inferior a la requerida para cubrir la canasta básica de alimentos de una familia de estrato socioeconómico bajo, que según el DANE era de de $\$ 762.917$ para el año 2004. Estos datos están de acuerdo con los reportes para las zonas de Urabá y el Bajo Cauca en 2005, cuando se encontró que $50 \%$ de los hogares tenían ingresos con un salario mínimo mensual o menos. Nuestros hallazgos son perfectamente compatibles con lo encontrado en el departamento de Antioquia, donde en 2005 , el $72 \%$ de los hogares se percibieron en inseguridad alimentaria, con mayores prevalencias en los hogares rurales y en el oriente (85\%), Bajo Cauca (83\%) y Urabá (78\%). En Urabá, 5\% de las familias urbanas y $2 \%$ de las rurales padece de inseguridad alimentaria con hambre grave, en tanto que en el Bajo Cauca $11 \%$ de las familias rurales y $8,5 \%$ de las urbanas padece de inseguridad alimentaria con hambre grave $(6,7)$.

En conclusión, la prevalencia de desnutrición en los niños de Turbo y El Bagre fue mayor que la informada para todo el departamento de Antioquia y el promedio nacional y se encontró asociación entre desnutrición crónica y carencia de servicios sanitarios y de agua potable, así como entre desnutrición aguda y crónica con los indicadores de hacinamiento.

\section{Agradecimientos}

A las comunidades de El Bagre y de Turbo, a los directivos y personal de los hospitales de Turbo y El Bagre y sus puestos de malaria; igualmente a los profesionales Alexandra Ríos, Juan Gabriel Piñeros, Tania Álvarez, José Nicolás Martínez y Patricia Rocha, quienes nos apoyaron en el trabajo de campo. A la nutricionista María Teresa Restrepo por su asesoría; a la Dirección Seccional de Salud de Antioquia y a la Universidad de Antioquia. 


\section{Conflicto de intereses}

Los autores manifiestan que no existe ningún conflicto de interés.

\section{Financiación}

El estudio fue financiado por la Dirección Seccional de Salud de Antioquia y la Universidad de Antioquia, convenio interadministrativo $\mathrm{Cl}-021$ 2004.

\section{Referencias}

1. Carmona-Fonseca J. Malaria, desnutrición y parasitosis intestinal en los niños colombianos: interrelaciones. latreia. 2004;17:354-69.

2. Jaramillo JA, Restrepo S. Hambre e inseguridad alimentaria, fracaso de las políticas contra el hambre. Perspectivas en Nutrición Humana. 2003;9:88-98.

3. Valdés-García LE. Pobreza y enfermedades emergentes y reemergentes. Medisan. 2000;4:39-50.

4. Carmona-Fonseca J. La malaria en Colombia, Antioquia y las zonas de Urabá y Bajo Cauca: panorama para interpretar la respuesta terapéutica antimalárica. Parte 1. latreia. 2003;16:299-318.

5. Carmona-Fonseca J. La malaria en Colombia, Antioquia y las zonas de Urabá y Bajo Cauca: panorama para interpretar la respuesta terapéutica antimalárica. Parte 2. latreia. 2004;17:34-53.

6. Álvarez MC, Benjumea MV, Roldán P, Maya M, Montoya EC. Perfil alimentario y nutricional de los hogares de la región del Bajo Cauca antioqueño. Medellín: Gobernación de Antioquia, Dirección Seccional de Salud, Programa de Mejoramiento Alimentario y Nutricional de Antioquia, Universidad de Antioquia, Escuela de Nutrición y Dietética; 2005.

7. Álvarez MC, Benjumea MV, Roldán P, Maya M, Montoya EC. Perfil alimentario y nutricional de los hogares de la región del Urabá antioqueño. Medellín: Gobernación de Antioquia, Dirección Seccional de Salud, Programa de Mejoramiento Alimentario y Nutricional de Antioquia, Universidad de Antioquia, Escuela de Nutrición y Dietética; 2005.

8. Ojeda G, Ordóñez M, Ochoa LH. Salud sexual y reproductiva en Colombia. Encuesta Nacional de Demografía y Salud 2005. Bogotá: Profamilia, ICBF, USAID, Ministerio de la Protección Social de Colombia, UNFPA; 2005. p.266-77.

9. Blair S, Carmona J, Correa AM. Malaria en niños: relaciones entre nutrición e inmunidad. Rev Panam Salud Pública. 2002;11:5-14.

10. Blair S, Álvarez G, Villa A, Carmona-Fonseca J, Ríos L. Estado nutricional y niveles de inmunoglobulinas y citoquinas en niños con malaria. Anales de Pediatría 2003;58:418-24.
11. Restrepo MT. Estado nutricional y crecimiento físico. Primera edición. Medellín: Universidad de Antioquia; 2000.

12. World Health Organization. Severe falciparum malaria. Trans R Soc Trop Med Hyg. 2000;94(Suppl. 1):S1-90.

13. Lohman TG, Roche AF, Martorell R. Anthropometric standardization reference manual. Champaign: Human Kinetics Books; 1988.

14. Organización Mundial de la Salud. El estado físico: uso e interpretación de la antropometría. Serie Informes Técnicos No. 854. Ginebra: OMS; 1995.

15. Ulijaszek SJ. Between-population variation in preadolescent growth. Eur J Clin Nutr. 1994;48(Suppl.1): S5-13.

16. De Onis M. Medición del estado nutricional con relación a la mortalidad. Bol Méd Hosp Infant Mex. 2002;59:70-5.

17. Pelletier DL. The relationship between child anthropometry and mortality in developing countries: implications for policy, programs and future research. J Nutr. 1994;124(Suppl.10):2047S-81.

18. Pelletier DL, Frongillo EA Jr, Habicht JP. Epidemiologic evidence for a potentiating effect of malnutrition on child mortality. Am J Public Health. 1993;83:1130-3.

19. Ruiz M, Ruiz N, Granados E, Peña LB. La mortalidad por desnutrición en Colombia. [Consultado: 31 de agosto de 2006]. Disponible en: http:// www.unipiloto.edu.co/files/resourcesmodule/ @random44edc86d04c8d/1180563214_mortalidad.pdf.

20. Gracia B, Plata C, Rueda A, Pradilla A. Antropometría por edad, género y estrato socioeconómico de la población escolarizada de la zona urbana de Cali. Colombia Médica. 2003;34:61-8.

21. Álvarez MC, Benjumea MV, Roldán P, Maya M, Montoya EC. Perfil alimentario y nutricional de los hogares antioqueños: análisis comparativo de algunos indicadores entre las regiones de Antioquia. Medellín: Gobernación de Antioquia, Dirección Seccional de Salud, Programa de Mejoramiento Alimentario y Nutricional de Antioquia, Universidad de Antioquia, Escuela de Nutrición y Dietética; 2005.

22. Dirección Seccional de Salud de Antioquia. Primeras causas de mortalidad agrupadas a 103 causas. Antioquia por municipios 2004. [Consultado: 25 de julio de 2007]. Disponible en: http://dssa.gov.co/ dowload/MORTALIDAD/10mortgeneral2004.xls.

23. Spurr GB, Barac-Nieto M, Maksud MG. Efficiency and daily work effort in sugar cane cutters. $\mathrm{Br} \mathrm{J}$ Ind Med. 1977;34:137-41.

24. Spurr GB, Maksud MG, Barac-Nieto M. Energy expenditure, productivity, and physical work capacity of sugarcane loaders. Am J Clin Nutr. 1977;30:1740-6. 
25. Spurr GB, Reina JC. Daily pattern of \%VO2max and heart rates in normal and undernourished school children. Med Sci Sports Exerc. 1990;22:643-52.

26. Núñez J, Cuesta L. Las trampas de pobreza en Colombia; ¿qué hacer? Diseño de un programa contra la extrema pobreza. Documento CEDE 2006-19. ISSN 1657-7191 (Edición electrónica). Abril de 2006. [Consultado: 25 de julio de 2007]. Disponible en: http:// economia.uniandes.edu.co/var/rw/archivos/cede/ documentos/d2006-19.pdf.

27. Stettler N, Schutz Y, Whitehead R, Jequier E. Effect of malaria and fever on energy metabolism in Gambian children. Pediatr Res. 1992;31:102-6.

28. Berclaz PY, Benedek C, Jequier E, Schutz Y. Changes in protein turnover and resting energy expenditure after treatment of malaria in Gambian children. Pediatr Res. 1996;39:401-9.

29. O'Donnell A, Weatherall DJ, Taylor AM, Reeder JC, Allen SJ. Muscle cell injury, haemolysis and dark urine in children with falciparum malaria in Papua New Guinea. Trans R Soc Trop Med Hyg. 2006;100:81725.

30. Friedman JF, Kwena AM, Mirel LB, Kariuki SK, Terlouw DJ, Phillips-Howard PA et al. Malaria and nutritional status among pre-school children: results from cross-sectional surveys in western Kenya. Am J Trop Med Hyg. 2005;73:698-704.

31. Cuartas G. Educación rural en Colombia. [Consultado: 25 de julio de 2007]. Disponible en: http://www. gloriacuartas.com/article-imprim.php3? id_article=9.
32. Contraloría General de la República. Evaluación de la Política Social 2003. Bogotá, marzo de 2004. [Consultado: 25 julio de 2007]. Disponible en: http:// www.contraloriagen.gov.co:8081/internet/html/ publicaciones/detalles.jsp?id=81

33. Vélez M. La obesidad de los gringos y la desnutrición en el resto del mundo. [Consultado: 8 de agosto de 2006]. Disponible en: http://deslinde.org.co/Ds/32/ La_obesidad_de_los_gringos_y_la_desnutrición_en_el_ resto_del_mundo.htm.

34. CEPAL. Los desplazamientos forzados. Serie Población y Desarrollo № 48. [Consultado: 25 de julio de 2007]. Disponible en: http://www.eclac.cl/ publicaciones/xml/0/14000/lcl2013-P3.pdf

35. Departamento Administrativo Nacional de Estadística. Censo General 2005. Censo Ampliado. [Consultado: 25 de julio de 2007]. Disponible en: http:// w w w.dane.gov.co/files/censo2005/ censo_ampliado.pdf.

36. Organización Panamericana de la Salud. Perfil de salud por país. Datos actualizados para 2001. Colombia. [Consultado: 8 de agosto de 2006] Disponible en: http:/ /www.paho.org/spanish/sha/prflcol.htm 2002.

37. Heineck G. Height and weight in Germany, evidence from the German Socio-Economic Panel, 2002. Econ Hum Biol. 2006;4:359-82.

38. Joshi N, Rikimaru T, Pandey S. Effects of economic status and education level on the height and weight of community adolescents in Nepal. J Nutr Sci Vitaminol (Tokyo). 2005;51:231-8. 